\title{
Kryolipolyse für submentales Fett und kleinere Problemzonen
}

Kleinere Fettansammlungen am Körper sind oft die am schwersten behandelbaren. Das Doppelkinn ist ein Ergebnis des Lebenswandels, des Alters oder der Genetik und kann - im Gegensatz zu vielen anderen Problemzonen - nicht versteckt werden. Eine Studie der ASDS (American Society for Dermatologic Surgery) aus dem Jahr 2014 erwies, dass ungefähr $68 \%$ der Befragten über unerwünschtes Fett am Kinn und im Nacken besorgt sind.

Der neue CoolMini ${ }^{\mathrm{T}}$ Applikator ist optimal zur Fettreduktion am Doppelkinn sowie an kleineren Problemzonen wie den Knien oder Achseln geeignet. Das Design des Applikators ist in seiner Grö- ße, Wölbung und Form ideal, um sich individuell an die Gesichtsform des Patienten und den sensiblen Bereich unter dem Kinn anzupassen. Im Schnitt benötigen die Patienten 1-2 Behandlungen, um das gewünschte Ergebnis zu erreichen.

Die ersten Resultate sind bereits nach 3 Wochen sichtbar, das Endergebnis ist nach 1-3 Monaten erreicht. Die Zulassung der US Food and Drug Administration (FDA) zur Behandlung des submentalen Fetts basiert auf den Daten einer US-Studie mit 60 Probanden/innen zwischen 22 und 65 Jahren. Im Rahmen der Studie erhielten die Patienten 1-2 Behandlungen unter dem Kinn im Zeit-
Mit dem neuen Applikator können auch kleinere Areale mittels Kryolipolyse behandelt werden.

raum von 6 Wochen. Es wurde eine deutliche Fettzellenreduktion im ähnlichen Ausmaß wie bei anderen CoolSculpting ${ }^{\oplus}$ Applikatoren festgestellt. Die Probanden/innen zeigten keine oder nur minimale Nebenwirkungen und keine Ausfallzeit.

Nach Informationen von Zeltiq Aesthetics

\section{Tumornekrosefaktor-Blocker erweitert Behandlungsspektrum}

Acne Inversa (AI), auch Hidradenitis suppurativa (HS) genannt, ist eine chronische Erkrankung, die typischerweise mit entzündlichen Hautarealen im Bereich der Achselhöhlen, der Leiste und am Gesäß einhergeht. Bis der Patient nach dem Auftreten der ersten Symptome die richtige Diagnose und eine adäquate Behandlung erhält, können mehrere Jahre vergehen, so Prof. Dr. Gregor Jemec, Kopenhagen, Dänemark. Mit der AI einhergehende Komorbiditäten sind u.a. Depression und Gelenkerkrankungen. Des Weiteren scheint eine Assoziation zwischen AI und Übergewicht, Rauchen, Hypertriglyzeridämie, niedrigem HDL, Diabetes und dem metabolischen Syndrom zu bestehen. In einer aktuellen Studie belegen Yadav S und Kollegen zudem bei Patienten mit entzündlichen Darmerkrankungen eine nahezu 9-fach erhöhte Wahrscheinlichkeit, auch eine HS zu entwickeln, so Jemec.

Eine HS-Behandlung sollte antiinflammatorisch, antifibrotisch, antibakteriell, schmerz- und juckreizlindernd und im besten Fall auch von Vorteil für die Behandlung assoziierter Erkrankungen sein, forderte Prof. Dr. Ulrich Mrowietz, Universitätshautklinik Kiel. Die Behandlung orientiere sich an den Vorgaben der aktuellen europäischen S1-Leitlinie zur Behandlung von HS/AI (Zouboulis et al. JEADV 2015;29:619-44).

Mit Adalimumab (Humira ${ }^{\bowtie}$ ) steht seit Ende Juli 2015 ein in der Europäischen Union (EU) zugelassenes Medikament zur Behandlung der AI zur Verfügung. Adalimumab ist indiziert bei erwachsenen Patienten mit aktiver mittelschwerer bis schwerer AI, die unzureichend auf konventionelle systemische Therapien angesprochen haben.

Die Zulassungserweiterung für Adalimumab für die Behandlung der AI basiert auf zwei Phase-III-Studien, PIONEER I und II. Primärer Endpunkt in beiden Studien war der Anteil an Patienten, die ein klinisches Ansprechen gemessen am HiSCR (Hidradenitis Suppurativa Clinical Response) erreichten. Dieser ist definiert als eine mindestens $50 \%$ ige Abnahme der Gesamtzahl der Abszesse und entzündlichen Knoten gegenüber dem Ausgangswert, wobei die
Anzahl der Abszesse oder dränierenden Fisteln nicht zunehmen darf.

Die Daten zeigen, dass bei Patienten mit mittelschwerer bis schwerer AI, die mit $40 \mathrm{mg} /$ Woche Adalimumab ab Woche 4 behandelt wurden (nach Gabe einer Initialdosis von $160 \mathrm{mg}$ Adalimumab in Woche 0 und $80 \mathrm{mg}$ in Woche 2), in Woche 12 signifikant höhere Ansprechraten erreicht wurden als in der Placebogruppe. In PIONEER II wurde bei nahezu jedem dritten Patient unter Adalimumab $(58,9 \%) \mathrm{zu}$ Woche 12 der primäre Endpunkt erreicht, gemessen am HiSCR, versus $27,6 \%$ unter Placebo. Darüber hinaus wurde in PIONEER II in Woche 12 bei einem signifikant höheren Anteil an Adalimumab-Patienten eine klinisch relevante Reduktion der Hautschmerzen (gemessen an einer $\geq 30 \%$ igen Reduktion von Hautschmerzen) gegenüber Placebo-Patienten erreicht (45,7 vs. $20,7 \%$, p < 0,001).

In PIONEER I und PIONEER II wurden keine neuen Sicherheitssignale identifiziert. Das Sicherheitsprofil bei Patienten mit AI entsprach somit dem bekannten Sicherheitsprofil von Adalimumab, so Mrowietz.

Ingo Schroeder

Satellitensymposium „PIONEERing a new frontier in $\mathrm{HS}^{\prime \prime}$ anlässlich des 24. Kongresses der European Academy of Dermatology and Venereology am 10.10.2015 in Kopenhagen, Dänemark. Veranstalter: AbbVie 